Revista de Matemática: Teoría y Aplicaciones 2006 13(2) : 117-124

CIMPA - UCR - CCSS ISSN: 1409-2433

\title{
UNA CARACTERIZACIÓN SOBRE LAS SOLUCIONES PARA EL PROBLEMA DE REAPROVISIONAMIENTO CONJUNTO
}

\author{
Sergio de-los-Cobos-Silva* Miguel A. Gutiérrez-Andrade ${ }^{\dagger}$ \\ John Goddard-Close Blanca Rosa Pérez-Salvador $^{\S}$
}

Recibido/Received: 22/02/06 - Aceptado/Accepted: 08/09/06

\begin{abstract}
Resumen
En este trabajo se presenta una introducción sobre el problema de inventario multiartículo conocido como el problema de reaprovisionamiento conjunto (JRP, del inglés Joint Replenishment Problem), el cual ha sido ampliamente investigado, puesto que se utiliza con mucha frecuencia en problemas reales. El problema JRP tiene una variable de decisión de tipo continua y tantas variables de decisión de tipo discretas como el número de artículos que se ordenan y producen. Para este problema existe un método exacto proporcionado por Goyal, que para un número grande de variables de decisión discretas lo vuelve prohibitivo. Se presenta una caracterización alternativa de las soluciones de tipo combinatorio para el problema de reaprovisionamiento conjunto.
\end{abstract}

Palabras clave: Problemas de inventarios multiartículo, problema de reaprovisionamiento conjunto.

\begin{abstract}
In this paper an introduction is given to a multiple-item inventory problem, known as the Joint Replenishment Problem (JRP). This is a real world problem which has been extensively studied. The JRP has a continuous decision variable, and as many discrete decision variables as products that are ordered and produced. An exact method, given by Goyal, exists for solving the JRP, however for a large number of
\end{abstract}

${ }^{*}$ Departamento de Ingeniería Eléctrica, Universidad Autónonoma Metropolitana - Iztapalapa, Av. San Rafael Atlixco No. 186, Col. Vicentina, Del. Iztapalapa, México D.F., C.P. 09340 México; Fax: +(52)(55) 58.04.46.40, México. E-Mail: cobos@xanum.uam.mx

${ }^{\dagger}$ Same address as S. de los Cobos. E-Mail: gamma@xanum.uam.mx

${ }^{\ddagger}$ Same address as S. de los Cobos. E-Mail: jgc@xanum.uam.mx

${ }^{\S}$ Departamento de Matemáticas, same address at the Universidad Autónoma Metropolitana. E-Mail: psbr@xanum.uam.mx 
118 S. De los Cobos-M. GutiéRreZ-J. GoddARD-B.PÉREZ Rev.Mate.Teor.Aplic. (2006) 13(2)

discrete decision variables the computational time required becomes prohibitive. Here, an alternative characterization of the solution for the combinatorial part of the JRP is given.

Keywords: Inventory mutiple-item problems, joint replenishment problem.

Mathematics Subject Classification: 90B05, 90C11.

\section{Introducción}

En los sistemas de inventarios multiartículo, el concepto de reaprovisionamiento (replenishment en inglés) se genera en alguna de las siguientes situaciones: a) cuando un artículo se empaqueta en más de un tipo de presentación inmediatamente después de su manufactura, b) cuando los artículos son ordenados de manera conjunta ó c) cuando varios artículos serán entregados por el mismo distribuidor. Una peculiaridad de los sistemas multiartículo es que usualmente se caracterizan por la existencia de alguna interacción entre los artículos, así por ejemplo, existe una interacción en el costo por ordenar cuando éstos son ordenados de manera conjunta, en estos casos se supone que es mayor el costo de los artículos cuando son ordenados separadamente que cuando son reaprovisionados de manera conjunta. El problema de reaprovisionamiento conjunto (JRP) es de gran aplicación en el mundo real, por lo que ha sido ampliamente investigado desde hace ya tiempo.

\section{El problema de reaprovisionamiento conjunto}

El JRP es un problema famoso en el mundo real y ocurre en diferentes situaciones, como se ha mencionado anteriormente. La ventaja económica de manufacturar artículos conjuntamente y empaquetarlos individualmente, se debe al hecho de que sí esos artículos son conjuntamente reaprovisionados (manufacturados y empaquetados) de manera individual entonces cada artículo contabiliza para el costo total de dos maneras: por su manufactura global y por el empaquetamiento individual.

Ahora bien, una política de empaquetar todos los artículos, una vez manufacturados, puede ser no económica puesto que empaquetar ciertos artículos tiene una economía de menor frecuencia que otros. En los sistemas de inventarios de reaprovisionamiento conjunto, el problema esencial es determinar la frecuencia de empaquetamiento óptima $K_{i}$ de los artículos de manera individual. El artículo con la mayor frecuencia de empaquetamiento, proporciona la frecuencia de manufactura óptima $T$ de los artículos. Los intervalos de reaprovisionamiento para cada artículo es por tanto un múltiplo del ciclo básico, es decir, $T_{i}=K_{i} T, \forall i=1,2,3, \ldots, n$, donde $n$ es el número de artículos diferentes y $K_{i}$ es un entero positivo.

Lo anterior, proporciona la visión del problema de determinar la frecuencia de empaquetamiento óptimo de los artículos reaprovisionados conjuntamente que es semejante al problema de determinar las cantidades de ordenamiento económico de los artículos proporcionados por un solo distribuidor (por ejemplo, artículos deshidratados en diferentes 
presentaciones, 50 grs., $1 / 2 \mathrm{~kg}$., $1 \mathrm{~kg}$., etc.), en tales sistemas de inventarios, el costo de poner una orden de compra para cierta cantidad de artículos consiste de dos componentes:

1. Un costo fijo, que es independiente de la cantidad de artículos ordenados, y

2. un costo variable, que depende de la cantidad de artículos que son ordenados.

Se puede ver la siguiente relación: el primer componente del costo de ordenamiento es semejante al costo de manufactura multiartículo y el segundo elemento del costo de ordenamiento es similar al costo de empaquetamiento.

En general el JRP es un problema de tipo entero mixto, por lo que se trata tanto de un problema combinatorio como de un problema de tipo continuo, donde lo que se desea es determinar cuándo y cuánto ordenar de cada uno de los artículos que se controlan.

Los supuestos considerados en este trabajo, se retoman de [2] y en [5] y son:

1. La demanda anual de cada artículo es conocida y constante.

2. El costo unitario en el tiempo permitido para reaprovisionamiento es conocido y constante.

3. No se permite déficit.

4. El horizonte de tiempo es infinito.

5. Las cantidades enteras de los artículos a reaprovisionarse se obtienen todas a la vez.

La siguiente nomenclatura es usual:

- $j$ : índice del artículo, $j=1,2,3, \ldots, n$.

- $D_{j}$ : Demanda anual del artículo $j$.

- $h_{j}$ : Costo anual de almacenamiento por unidad del artículo $j$.

- $S$ : Costo mayor por cada orden asociado con un reaprovisionamiento que es independiente del tipo de los artículos o del número de éstos.

- $S_{j}$ : Costo menor por ordenamiento del artículo $j$ cuando se incluye en un grupo de reaprovisionamiento(adicional a $S$ ).

- $T$ : Ciclo de tiempo básico.

- $K_{j}$ : Número entero que indica la frecuencia(número de ciclos) en la cual el artículo $j$ es ordenado.

- $C$ : Costo total promedio anual, el cual se compone de los costos de ordenación y almacenamiento.

- Obsérvese que las constantes:

$$
S, h_{j}, D_{j}, S_{j}, \forall j=1,2,3, \ldots, n,
$$

son estrictamente mayores que cero. 
El costo total $C$ está en función de $n+1$ variables de decisión, de las cuales una es de tipo contínuo $T$, y las demás $K_{i}, i=1,2,3, \ldots, n$ son de tipo entero. Se puede observar que el problema es el encontrar un mínimo global, ya que, la función tiene muchos puntos que son mínimos locales, puesto que la función no es conjuntamente convexa respecto de $T$ y $K_{i}, \forall i=1,2, \ldots, n$. Definamos $N$ como el inverso multiplicativo de $T$, es decir, $N=\frac{1}{T}$, entonces se tiene que el costo total promedio anual está dado por:

$$
C\left(N, K_{1}, K_{2}, K_{3}, \ldots, K_{n}\right)=N\left(S+\sum_{j=1}^{n} \frac{S_{j}}{K_{j}}\right)+\frac{1}{2 N} \sum_{j=1}^{n} h_{j} D_{j} K_{j} .
$$

Dada una solución combinatoria $K=\left(K_{1}, K_{2}, \ldots, K_{n}\right)$, se puede obtener la solución continua $T$ derivando respecto de $N$ e igualando a cero, es decir:

$$
0=\frac{\partial C\left(N, K_{1}, K_{2}, K_{3}, \ldots, K_{n}\right)}{\partial N}=\left(S+\sum_{j=1}^{n} \frac{S_{j}}{K_{j}}\right)-\frac{1}{2 N^{2}} \sum_{j=1}^{n} h_{j} D_{j} K_{j}
$$

obteniéndose que:

$$
N^{*}(K)=\left(\frac{\sum_{j} h_{j} D_{j} K_{j}}{2\left(S+\sum_{j} \frac{S_{j}}{K_{j}}\right)}\right)^{\frac{1}{2}} .
$$

Similarmente, para un valor particular $T_{0}$ de $T$, en [2] se puede observar que la correspondiente solución combinatoria óptima $K=\left(K_{1}, K_{2}, \ldots, K_{n}\right)$ debe satisfacer que:

$$
K_{j}\left(K_{j}-1\right)<\frac{2 S_{j}}{T_{0}^{2} \alpha_{j}} \leq K_{j}\left(K_{j}+1\right), \forall j=1,2, \ldots, n,
$$

donde $\alpha_{j}=h_{j} D_{j}, \forall j=1,2, \ldots, n$.

Ahora bien, para un valor $N^{*}(K)$ suponga que existen dos soluciones combinatorias diferentes, digamos $K^{*}=\left(K_{11}, K_{12}, K_{13}, \ldots, K_{1 n}\right)$ y $K^{* *}=\left(K_{21}, K_{22}, K_{23}, \ldots, K_{2 n}\right)$, esto significa que existe al menos una $j \in\{1,2,3, \ldots, n\}$ tal que $K_{1 j} \neq K_{2 j}$ para las que se cumple que $N^{*}\left(K^{*}\right)=N^{*}\left(K^{* *}\right)$, por lo que considerando la ecuación 2 se tiene que:

$$
N^{*}\left(K^{*}\right)=\left(\frac{\sum_{j=1}^{n} h_{j} D_{j} K_{1 j}}{2\left(S+\sum_{j=1}^{n} \frac{S_{j}}{K_{1 j}}\right)}\right)^{\frac{1}{2}}=\left(\frac{\sum_{j=1}^{n} h_{j} D_{j} K_{2 j}}{2\left(S+\sum_{j=1}^{n} \frac{S_{j}}{K_{2 j}}\right)}\right)^{\frac{1}{2}}=N^{*}\left(K^{* *}\right) .
$$

es decir, se debe cumplir que:

$$
\left(S+\sum_{j=1}^{n} \frac{S_{j}}{K_{1 j}}\right)\left(\sum_{j=1}^{n} \alpha_{j} K_{2 j}\right)=\left(S+\sum_{j=1}^{n} \frac{S_{j}}{K_{2 j}}\right)\left(\sum_{j=1}^{n} \alpha_{j} K_{1 j}\right) .
$$

Sean los conjuntos $w=\left\{z \mid K_{1 z} \neq K_{2 z}, z=1,2, \ldots, n\right\}$, con cardinalidad $\|w\|=l \mathrm{y}$ su complemento $w^{c}=\left\{i \mid K_{1 i}=K_{2 i}, i=1,2, \ldots, n\right\}$, con cardinalidad $\left\|w^{c}\right\|=n-l$. Sea 
$\alpha_{j}=h_{j} D_{j}, \forall j=1,2,3, \ldots, n$, entonces se dirá que, dadas dos soluciones combinatorias $K^{*}$ y $K^{* *}$ para una misma solución continua $N^{*}$, es decir, $N^{*}\left(K^{*}\right)=N^{*}\left(K^{* *}\right), K^{*}$ domina a $K^{* *}$ en orden $m$, si y sólo si, $\|w\|=m, K_{1 z}>K_{2 z}, \forall z \in w$. Si $K^{*}$ domina a $K^{* *}$ en todo orden $l \in\{1,2, \ldots, n\}$, entonces se dirá que $K^{*}$ domina absolutamente a $K^{* *}$. De lo anterior se tienen las siguientes igualdades:

$$
\begin{gathered}
\sum_{j=1}^{n} K_{j}=\sum_{z \in w} K_{z}+\sum_{i \in w^{c}} K_{i} \\
\sum_{j=1}^{n} \alpha_{j} K_{j}=\sum_{z \in w} \alpha_{z} K_{z}+\sum_{i \in w^{c}} \alpha_{i} K_{i}, \\
\sum_{j=1}^{n} \frac{S_{j}}{K_{j}}=\sum_{z \in w} \frac{S_{z}}{K_{z}}+\sum_{i \in w^{c}} \frac{S_{i}}{K_{i}},
\end{gathered}
$$

Además se tiene que:

$$
\begin{gathered}
\sum_{j=1}^{n} K_{1 j}=\sum_{z \in w} K_{1 z}+\sum_{i \in w^{c}} K_{1 i}=\sum_{z \in w} K_{1 z}+\sum_{i \in w^{c}} K_{2 i} \\
\sum_{j=1}^{n} \alpha_{j} K_{1 j}=\sum_{z \in w} \alpha_{z} K_{1 z}+\sum_{i \in w^{c}} \alpha_{i} K_{1 i}=\sum_{z \in w} \alpha_{z} K_{1 z}+\sum_{i \in w^{c}} \alpha_{i} K_{2 i} \\
\sum_{j=1}^{n} \frac{S_{j}}{K_{1 j}}=\sum_{z \in w} \frac{S_{z}}{K_{1 z}}+\sum_{i \in w^{c}} \frac{S_{i}}{K_{1 i}}=\sum_{z \in w} \frac{S_{z}}{K_{1 z}}+\sum_{i \in w^{c}} \frac{S_{i}}{K_{2 i}} .
\end{gathered}
$$

Substitutendo las ecuaciones 8, 9 y 10 en 4, se obtiene:

$$
\begin{aligned}
& \left(S+\left(\sum_{z \in w} \frac{S_{z}}{K_{1 z}}+\sum_{i \in w^{c}} \frac{S_{i}}{K_{2 i}}\right)\right)\left(\sum_{z \in w} \alpha_{z} K_{2 z}+\sum_{i \in w^{c}} \alpha_{i} K_{2 i}\right) \\
= & \left(S+\left(\sum_{z \in w} \frac{S_{z}}{K_{2 z}}+\sum_{i \in w^{c}} \frac{S_{i}}{K_{2 i}}\right)\right)\left(\sum_{z \in w} \alpha_{z} K_{1 z}+\sum_{i \in w^{c}} \alpha_{i} K_{2 i}\right) .
\end{aligned}
$$

de donde

$$
\begin{aligned}
& S \sum_{z \in w} \alpha_{z} K_{2 z}+\sum_{z \in w} \frac{S_{z}}{K_{1 z}}\left(\sum_{z \in w} \alpha_{z} K_{2 z}+\sum_{i \in w^{c}} \alpha_{i} K_{2 i}\right)= \\
& S \sum_{z \in w} \alpha_{z} K_{1 z}+\sum_{z \in w} \frac{S_{z}}{K_{2 z}}\left(\sum_{z \in w} \alpha_{z} K_{1 z}+\sum_{i \in w^{c}} \alpha_{i} K_{2 i}\right)
\end{aligned}
$$

Recuérdese que: $\sum_{i \in w^{c}} \alpha_{i} K_{1 i}=\sum_{i \in w^{c}} \alpha_{i} K_{2 i}$ y que: $\sum_{i \in w^{c}} \frac{S_{i}}{K_{1 i}}=\sum_{i \in w^{c}} \frac{S_{i}}{K_{2 i}}$, por lo que igualando a cero se tiene:

$$
S \sum_{z \in w} \alpha_{z} K_{1 z}+\sum_{z \in w} \frac{S_{z}}{K_{2 z}} \sum_{z \in w} \alpha_{z} K_{1 z}+\sum_{z \in w} \frac{S_{z}}{K_{2 z}} \sum_{i \in w^{c}} \alpha_{i} K_{2 i}
$$


122 S. De los Cobos-M. GutiÉRreZ-J. GoddARD-B.PÉREZ Rev.Mate.Teor.Aplic. (2006) 13(2)

$$
\begin{gathered}
+\sum_{i \in w^{c}} \frac{S_{i}}{K_{2 i}} \sum_{z \in w} \alpha_{z} K_{1 z}-S \sum_{z \in w} \alpha_{z} K_{2 z} \\
-\sum_{z \in w} \frac{S_{z}}{K_{1 z}} \sum_{z \in w} \alpha_{z} K_{2 z}-\sum_{z \in w} \frac{S_{z}}{K_{1 z}} \sum_{i \in w^{c}} \alpha_{i} K_{2 i} \\
-\sum_{i \in w^{c}} \frac{S_{i}}{K_{2 i}} \sum_{z \in w} \alpha_{z} K_{2 z}=0,
\end{gathered}
$$

de donde

$$
\begin{gathered}
S \sum_{z \in w} \alpha_{z} K_{1 z}+\sum_{z \in w} \frac{S_{z}}{K_{2 z}} \sum_{z \in w} \alpha_{z} K_{1 z}+\sum_{z \in w} \frac{S_{z}}{K_{2 z}} \sum_{i \in w^{c}} \alpha_{i} K_{2 i} \\
+\sum_{i \in w^{c}} \frac{S_{i}}{K_{2 i}} \sum_{z \in w} \alpha_{z} K_{1 z} \\
\left.-S \sum_{z \in w} \alpha_{z} K_{2 z}-\sum_{z \in w} \frac{S_{z}}{K_{1 z}} \sum_{z \in w} \alpha_{z} K_{2 z}\right)-\sum_{z \in w} \frac{S_{z}}{K_{1 z}} \sum_{i \in w^{c}} \alpha_{i} K_{2 i} \\
-\sum_{i \in w^{c}} \frac{S_{i}}{K_{2 i}} \sum_{z \in w} \alpha_{z} K_{2 z}=0,
\end{gathered}
$$

por tanto se tiene que:

$$
\begin{gathered}
S\left(\sum_{z \in w} \alpha_{z} K_{1 z}-\sum_{z \in w} \alpha_{z} K_{2 z}\right)+\sum_{i \in w^{c}} \frac{S_{i}}{K_{2 i}} \sum_{z \in w} \alpha_{z} K_{1 z}-\sum_{i \in w^{c}} \frac{S_{i}}{K_{2 i}} \sum_{z \in w} \alpha_{z} K_{2 z} \\
+\sum_{z \in w} \frac{S_{z}}{K_{2 z}} \sum_{j=1}^{n} \alpha_{j} K_{1 j}-\sum_{z \in w} \frac{S_{z}}{K_{1 z}} \sum_{j=1}^{n} \alpha_{j} K_{2 j}=0
\end{gathered}
$$

reacomodando se obtiene:

$$
\begin{aligned}
& S \sum_{z \in w} \alpha_{z}\left(K_{1 z}-K_{2 z}\right)+\sum_{i \in w^{c}} \frac{S_{i}}{K_{2 i}} \sum_{z \in w} \alpha_{z}\left(K_{1 z}-K_{2 z}\right) \\
& +\left(\sum_{z \in w} \frac{S_{z}}{K_{2 z}} \sum_{j=1}^{n} \alpha_{j} K_{1 j}-\sum_{z \in w} \frac{S_{z}}{K_{1 z}} \sum_{j=1}^{n} \alpha_{j} K_{2 j}\right)=0 \\
\Longrightarrow & \left(S+\sum_{i \in w^{c}} \frac{S_{i}}{K_{2 i}}\right) \sum_{z \in w} \alpha_{z}\left(K_{1 z}-K_{2 z}\right)+ \\
+ & \left(\sum_{z \in w} \frac{S_{z}}{K_{2 z}} \sum_{j=1}^{n} \alpha_{j} K_{1 j}-\sum_{z \in w} \frac{S_{z}}{K_{1 z}} \sum_{j=1}^{n} \alpha_{j} K_{2 j}\right)=0
\end{aligned}
$$


Si $K^{*}$ domina a $K^{* *}$ en cualquier orden $l$ para $l \in\{1,2, \ldots, n\}$, entonces se cumple que:

$$
\begin{gathered}
\sum_{z \in w} \alpha_{z}\left(K_{1 z}-K_{2 z}\right)>0, \\
\sum_{z \in w} \frac{S_{z}}{K_{2 z}}>\sum_{z \in w} \frac{S_{z}}{K_{1 z}}, \\
\sum_{j=1}^{n} \alpha_{j} K_{1 j}>\sum_{j=1}^{n} \alpha_{j} K_{2 j} .
\end{gathered}
$$

lo que sería una contradicción en la ecuación (17) puesto que sería estrictamente positiva.

Por tanto, dada una solución continua $N^{*}$, no existen dos soluciones combinatorias $K^{*}$ y $K^{* *}$ tales que $N^{*}\left(K^{*}\right)=N^{*}\left(K^{* *}\right)$ y una domine a la otra en cualquier orden. Es más, dada una solución continua $N^{*}$ y dada una solución combinatoria con $n-1$ entradas fijas, existe una y sólo una solución para la n-ésima entrada.

\section{Conclusiones}

Aunque la caracterización de las soluciones que se han realizado en este trabajo es de tipo teórico, el tener en cuenta la idea de dominancia de soluciones en su implementación práctica puede ahorrar mucho esfuerzo computacional, principalmente en la utilización de heurísticas como es el caso del llamado algoritmo RAND [7] que se utiliza de manera usual, incluso para probar otros métodos. El JRP usualmente ha sido atacado mediante el encontrar los valores óptimos de $K_{i}, i=1,2,3, \ldots, n$ y después derivando para así encontrar el valor óptimo del ciclo básico $T$, este procedimiento a lo que ha conllevado es el de tratar básicamente al JRP como un problema de optimización combinatoria, por lo que el valor del presente trabajo radica en observar que, si bien existe una solución óptima combinatoria, también existe de manera respectiva una solución a un problema de tipo continuo, por lo que se puede tratar de encontrar primero la solución de éste y posteriormente encontrar los correspondientes valores combinatorios $K_{i}, i=1,2,3, \ldots, n$, bajo esta perspectiva, se pueden utilizar algunos métodos como sección dorada y posteriormente encontrar cual es el respectivo conjunto de $K_{i}, i=1,2,3, \ldots, n$. En algunos trabajos [4] se utilizó la idea de primero ajustar sobre una solución continua y posteriormente encontrar las soluciones combinatorias, observando que se encuentran mejores resultados o al menos iguales a aquellos que se pueden obtener por el método RAND. Además de los métodos exactos propuestos por Goyal [2] y Van Eijs [10], se tienen otros como en [11] y se han estado implementando otros métodos heurísticos aparte de los ya enunciados, como es el caso en [3] y [8], entre otros.

\section{Referencias}

[1] de-los-Cobos-Silva, S.G.; Goddard-Close, J.; Gutiérrez- Andrade, M.A.; PérezSalvador, B.R.; González-Santoyo, F. (2005) "A comparison of different heuristic 
124 S. De los Cobos-M. GutiérReZ-J. GoddARD-B.PÉREZ Rev.Mate.Teor.Aplic. (2006) 13(2)

methods applied to a mixed integer programming problem", J. Gil-Aluja, F. GonzálezSantoyo, B. Flores-Romero \& J. Flores-Romero J. (Eds.) Techniques and Methodologies for the Modeling and Simulation of Systems, AMSE: 214-219.

[2] Goyal, S. (1974) "Determination of optimum packaging frequency of items jointly replenished", Management Science 21(4): 436-443.

[3] Gutiérrez-Andrade, M.A.; de-los-Cobos-Silva, S.G.; Goddard, J. (2006) "A comparison between RAND and scatter search for solving the joint replenishment problem", XIII CLAIO - Congreso Latino-Iberoamericano de Investigación Operativa, Montevideo, Uruguay, November 27-28, 2006.

[4] Gutiérrez-Andrade, M.A.; de-los-Cobos-Silva, S.G.; Goddard, J. (2006) "Two new algorithms for the joint replenishment problem", VI PanAmerican Workshop, Oaxaca, México, June 23-28, 2006.

[5] Kaspi, M.; Rosenblatt, M. (1983) "An improvement of silver's algorithm for the joint replenishment problem", IIE Transactions 15: 264-269.

[6] Kaspi, M.; Rosenblatt, M. (1985) "The effectiveness of heuristic algoritms for multiitem inventory systems with joint replenishment costs", International Journal of Production Research 29(1): 107-114.

[7] Kaspi, M.; Rosenblatt, M. (1991) "On the economic ordering quantity for jointly replenishment items", International Journal of Production Research 29(1): 107-114.

[8] Khousa, M.; Michalewics, Z.; Sotoskar, S. (2000) "A comparison between genetic algorithms and the RAND method for solving the joint replenishment problem", Production Planning \& Control II(6): 556-564.

[9] Silver, A. (1976) "A simple method of determining order quantities in joint replenishments under deterministic demand", Management Science 22: 1351-1361.

[10] Van Eijs, M. J. (1993) "A note on the joint replenishment problem under constant demand", J. Opl. Res. Soc. 44(2): 185-191.

[11] Viswanathan, S. (1996) "A new optimal algorithm for the joint replenishment problem", J. Opl. Res. Soc. 47: 936-944. 